SUBJECT AREAS: ASSAY SYSTEMS

METABOLOMICS

MOLECULAR ENGINEERING IN

PLANTS

AGRICULTURAL GENETICS

SCIENTIFIC REPORTS:

$3: 3082$

DOI: $10.1038 /$ srep03082

(2013)

Published: 30 October 2013

Updated:

27 January 2014

\section{CORRIGENDUM: Assessment of Genetically Modified Soybean in Relation to Natural Variation in the Soybean Seed Metabolome}

\author{
Joseph D. Clarke, Danny C. Alexander, Dennis P. Ward, John A. Ryals, Matthew W. Mitchell, \\ Jacob E. Wulff \& Lining Guo
}

This Article contains errors arising from misuse of the terms "substantial equivalence" and "equivalence".

In the Abstract, "An important aspect of GM crop adoption is to demonstrate safety and equivalence with respect to conventional crops. Untargeted metabolomics has the ability to profile diverse classes of metabolites and thus could be an adjunct for GM crop substantial equivalence assessment." should read: "An important aspect of GM crop adoption is to demonstrate safety; identifying differences in end points with respect to conventional crops is a part of the safety assessment process. Untargeted metabolomics has the ability to profile diverse classes of metabolites and thus could be an adjunct for identification of differences between the GM crop and its conventional counterpart."

In the Introduction, "A broad variety of methods have been used in the assessment of safety and equivalence for GM crops, and regulatory agencies rightly demand that the most advanced and accurate available technologies be applied." should read: "A broad variety of methods and tests have been used to generate data that is used in the assessment of safety for the new GM crop, and regulatory agencies rightly demand that the most advanced and accurate available technologies be applied."

In the Discussion, "We propose that comparing a GM line to the range of performance in a crop's native germplasm pool by metabolomic analysis could be a useful and proper standard as part of a safety and equivalence assessment program." should read: "We propose that comparing a GM line to the range of performance in a crop's native germplasm pool by metabolomic analysis could be a useful and proper standard as part of a safety and metabolite characterization program." 